\title{
journal
}

\section{Do Grain Boundaries Affect Microwave Dielectric Loss in Oxides?}

\begin{tabular}{|r|l|}
\hline Journal: & Journal of the American Ceramic Society \\
\hline Manuscript ID: & JACERS-25261.R1 \\
\hline Manuscript Type: & Article \\
\hline Date Submitted by the \\
Author: & 04-Dec-2008 \\
\hline Complete List of Authors: & $\begin{array}{l}\text { Breeze, Jonathan; Imperial College London, Materials } \\
\text { Perkins, James; Imperial College London, Materials } \\
\text { McComb, David; Imperial College London, Materials } \\
\text { Alford, Neil; Imperial College London, Department of Materials }\end{array}$ \\
\hline Keywords: & \begin{tabular}{l} 
dielectric materials/properties, grain boundaries \\
\hline
\end{tabular} \\
\hline
\end{tabular}

\section{ScholarONE \\ Manuscript Central}




\title{
Do Grain Boundaries Affect Microwave Dielectric Loss in Oxides?
}

\author{
Jonathan D. Breeze, James M. Perkins, David W. McComb, Neil McN. Alford. \\ Department of Materials, Imperial College London, London, UK, SW7 2AZ.
}

\begin{abstract}
Reducing loss in microwave dielectrics is critical to improving performance in wireless communications systems. Grain boundaries in polycrystalline microwave dielectric ceramics have long been suspected of increasing dielectric loss. They are often cited as the main contributor to the observed difference in dielectric losses between single crystals and polycrystalline ceramics. The exact configuration of grain boundaries is problematic to quantify in practice and their influence on the dielectric loss difficult to distinguish from other defects such as porosity, oxygen vacancies, impurities and dislocations. Here we measure the sensitivity of a single grain boundary in a magnesium oxide bi-crystal to the polarisation of an applied microwave field as a function of temperature.
\end{abstract}

\footnotetext{
Acknowledgments The authors acknowledge the support of the Engineering and Physical Sciences Research Council.

Corresponding Author: Neil McN Alford n.alford@imperial.ac.uk
} 


\section{Introduction}

One question that has never been convincingly answered is whether grain boundaries in polycrystalline ceramics influence dielectric loss. In microwave filters and a range of communications devices, low dielectric loss is essential for good performance, so this question is an important one to address. Single crystals often exhibit superior properties in comparison with their polycrystalline analogues. Single crystal whiskers of alumina can approach their theoretical tensile strength of $40 \mathrm{GPa}$, approximately $10 \%$ of the Young Modulus $\mathrm{E}^{1}$, while the strength of polycrystalline alumina is usually around $0.4 \mathrm{GPa}$. In polycrystalline ceramic materials, strength is usually dictated by processing defects that act as critical flaws ${ }^{2}$, limiting tensile strength to around $0.1 \%$ of $\mathrm{E}$. When these processing defects are absent, strength is governed by the length of the largest grain boundaries ${ }^{3}$. The relation between electrical properties and grain size in electroceramics is not clear since there are conflicting views regarding the effect of grain size on microwave dielectric loss. The general opinion though is that grain boundaries do have an adverse effect on microwave dielectric loss. It is not surprising then, that grain boundaries have been held responsible for contributing to the dielectric loss in ceramic materials.

The huge differences in microstructure and perfection between single crystals and their polycrystalline analogues are clear indicators why the conventional wisdom has assumed the impossibility of achieving a dielectric loss in polycrystalline ceramics approaching that of single crystals. Earlier studies have demonstrated the deleterious effects of impurities and it is certainly true that even minor amounts of impurity will cause huge increases in dielectric loss ${ }^{4}$. The problem though, is that because impurities have such a major influence, it is difficult to isolate the effect of grain boundaries. This is particularly the case in polycrystalline ceramics where the sintering process sweeps impurities to grain boundaries so that impurities and grain boundaries are inextricably linked. In the work described below we restrict our analysis to a material with a low relative permittivity on the grounds that ferroelectric materials possess additional loss mechanisms that complicate the discussion. The experiment described is carried out on $\mathrm{MgO}$ with a relative permittivity of less than 10 . 
Dielectric loss is described by the imaginary part of the permittivity, or more commonly by its ratio to the real part, the loss tangent $\tan \delta$. The problem of dielectric loss at high frequency was studied by Lord Rayleigh who published a paper in 1897 on dielectric waveguides ${ }^{5}$ and in 1909 by Debye on the subject of dielectric spheres ${ }^{6}$. Losses in bulk crystals fall into two categories, intrinsic and extrinsic. Intrinsic losses are dependent on the crystal structure and can be described by relaxation of the non-equilibrium phonon distribution created by an alternating electric field ${ }^{7,8,9}$. These intrinsic losses set the lower limit of losses found in pure 'defect free' single crystals. Extrinsic losses are associated with imperfections in the crystal structure, e.g. impurities, microstructural defects, grain boundaries, porosity, microcracks and random crystallite orientation. It is clear from our experiments, and those of others, that the loss in sintered ceramics is limited by these extrinsic losses but the precise influence of grain boundaries has never been determined.

It seems reasonable to assume that if grain boundaries are a source of dielectric loss, then reducing their number might be expected to reduce the dielectric loss. The logical extension of this assumption is the case of single crystals that do indeed show very low loss ${ }^{7,10}$ but this assumption is incorrect. Ceramic materials have been observed to show grain size dependence but this relationship is not straightforward. Kim et al ${ }^{11}$, in a study of $\mathrm{Ni}$-doped $\mathrm{Ba}\left(\mathrm{Ni}_{1 / 3} \mathrm{Nb}_{2 / 3}\right) \mathrm{O}_{3}$ found that the $\tan \delta$ decreased as the grain size increased. However, the system was complicated by the fact that there were porosity variations, ordering parameter variations and the possible presence of a liquid phase associated with an increase in $\tan \delta$. Ichinose et a ${ }^{12}$ found that the $\tan \delta$ in $\mathrm{Ba}\left(\mathrm{Mg}_{1 / 3} \mathrm{Ta}_{2 / 3}\right) \mathrm{O}_{3}$ ceramics decreased as grain size increased, saturating at a grain size of around $9 \mu \mathrm{m}$. Kim et $a l^{13}$ found that ordering played a dominant role in the dielectric loss of $\mathrm{Ba}\left(\mathrm{Mg}_{1 / 3} \mathrm{Ta}_{2 / 3}\right) \mathrm{O}_{3}$ so that again, grain size effects were masked. However in the region where ordering was constant (at the lower sintering temperatures), they found an increase in $\tan \delta$ as grain size decreased. The problem with such perovskite systems is that the interplay of so many properties such as grain size, porosity, cation ordering and liquid phases makes it difficult to make definitive remarks on the relationship between grain size and loss. In a study of $\mathrm{BiVO}_{5}$, Prasad et $\mathrm{al}^{14}$ found that the dielectric constant and dielectric loss (at $100 \mathrm{kHz}$ ) increased with increasing grain size but the problem in this study was that the density also varied significantly making a firm correlation between grain size and dielectric properties unreliable. Chen et l $^{15}$ in 
a study of $\mathrm{Ca}\left(\mathrm{Zn}_{1 / 3} \mathrm{Nb}_{2 / 3}\right) \mathrm{O}_{3}$ ceramics found that the dielectric loss decreased as the grain size increased. This was attributed to a decrease in the length of grain boundaries and hence the lattice defects in the vicinity of the grain boundary. However, they also suggested that there was a greater oxygen deficiency in ceramics with larger grains. On annealing in oxygen an improvement in $Q$ factor of around $30 \%$ was observed. Unfortunately no evidence regarding the degree of oxygen deficiency or lattice defects was given. In $\mathrm{ZnO}$ doped $\mathrm{CaTi}_{1-x}\left(\mathrm{Fe}_{1 / 2} \mathrm{Nb}_{1 / 2}\right)_{x} \mathrm{O}_{3}$, Kucheiko et $a l^{16}$ found that the tan $\delta$ decreased with increasing grain size but in the undoped material the grain size did not influence tan $\delta$. In ferroelectric materials such as $\mathrm{BaTiO}_{3}$ the effect of grain size on ferroelectricity is rather complex but in general there is a decreased $\tan \delta$ with decreasing grain size, however there is a significant frequency dependence associated with the resonant frequency of the domains which masks the real relation between microwave loss and grain size. In $\mathrm{PbTiO}_{3}$ grain size plays an important role in governing domain switching, but again the correlation with grain size is complex ${ }^{17}$. Alford et al studied polycrystalline $\mathrm{Al}_{2} \mathrm{O}_{3}$, where the density remained constant at $98.1 \pm 0.5 \%$ of theoretical density and cation ordering, liquid phases and ferroelectricity were absent. Within the accuracy of the measurement the relative dielectric constant displayed no variation with grain size. The same was not true for the $\tan \delta$. It was expected that as the grain size increased, the $\tan \delta$ would decrease because a reduction in the number of grain boundaries per unit volume would result in a material with a lower loss. In fact the tan $\delta$ remained constant at approximately $2.5 \times 10^{-5}$ for samples with a grain size less than about $3 \mu \mathrm{m}$ and then increased linearly as grain size increased to a value of $10^{-4}$ at 7 $\mu \mathrm{m}$. In a theoretical treatment, Schlomann ${ }^{18}$ analysed the losses expected in ionic crystals with disordered charge distributions and concluded that their contribution to loss (in magnetic spinels at least) would be negligible unless the grain size was extremely small. He assumed a very small average grain size of $0.01 \mu \mathrm{m}$ suggesting that most ceramic materials would not be affected by these losses. This is highly likely on the grounds that the average grain size in microwave dielectric ceramics is usually much greater than $0.01 \mu \mathrm{m}$. 


\section{Experimental Procedure}

\section{(1) Microscopy}

We examined the microstructure and electrical characteristics, (relative permittivity and the tan $\delta$ as a function of temperature) of $\mathrm{MgO}$ single crystal and bi-crystal spheres of $10 \mathrm{~mm}$ diameter. Single crystal spheres of $\mathrm{MgO}$ were cut from a large single bi-crystal (PDCrystals, Netherlands) grown by an arc-image furnace technique. The cut was arranged such that some of the spheres possessed a single grain boundary through their equator and some possessed no grain boundary. In order to examine the microstructure of the grain boundary we sliced the $\mathrm{MgO}$ sphere using a diamond saw and polished the face. The grain boundary was examined by polarised light optical microscopy and by transmission electron microscopy (FEI Titan 80/300).

\section{(2) Electrical Measurements}

For the electrical characterisation we used a dielectric resonator technique where a $\mathrm{MgO}$ sphere is placed inside a cylindrical silver-plated brass cavity on a low-loss single crystal quartz ring support structure. The MgO sphere was held in place by a thin PTFE piston rod which was secured by a steel spring as shown in Figure 1.

Coupling microwave energy into the sphere was achieved via two antenna loops protruding from the side of the cavity and oriented so as to excite or probe an axial magnetic field component. The conductivity of the silver plated cavity was characterised by measuring the unloaded quality factor $Q_{0}$ of the $\mathrm{TE}_{011}$ mode in the empty cylindrical cavity as a function of temperature. The conductivity of the cavity at room temperature was $5.94 \times 10^{7} \Omega^{-1} \mathrm{~m}^{-1}$. The diameter $D$ of the cylindrical cavity was $24.00 \mathrm{~mm}$ and its height $H$ was $16.10 \mathrm{~mm}$. The $\mathrm{TE}_{01 \delta}$ fundamental mode was used due to its high electric energy filling factor, low conductor losses and axisymmetric field distribution. Also, the effect of the PTFE rod could be neglected due to the very small electric filling factor along the cavity axis. The use of this mode allows the electric field to be parallel or normal to the grain boundary when the sphere is aligned vertically or horizontally with respect to the cavity axis. The quartz was cut (001) so that the axial component of its uniaxial 
anisotropic permittivity was commensurate with the cavity. Transmission measurements of the resonant frequency and quality factor were performed on an Agilent HP8720C Vector network analyser with $1 \mathrm{~Hz}$ resolution. The measurements were performed over the temperature range $25-300 \mathrm{~K}$ by placing the cavity on the cold head of a closed cycle Gifford McMahon cooler (Cryophysics).

\section{Results and Discussion}

\section{(1) Transmission Electron Microscopy}

The grain boundary is seen in fig 2, a high resolution TEM photo of the boundary. It is a remarkably clean interface and there is no evidence of a second phase or amorphous phase at the grain boundary.

\section{(2) Electrical Measurements}

A multi-region, multi-layer radial mode matching technique for axisymmetric cylindrical structures $^{19,20}$ was used to calculate the relative permittivity $\varepsilon_{r}$ and loss tangent tan $\delta$ using the measured data. A linear system of equations can be solved for the unknown permittivity given the resonant frequency. For axisymmetric cylindrical modes, the problem of modelling the resonator is reduced to a two dimensional one. Unfortunately, the stair-casing problem occurs when attempting to model the curved surfaces of a sphere using rectilinear regions. To overcome this, a method suggested by Hui ${ }^{21}$ was used where the average results were taken for two approximations, where the stepped outline of the rectilinear approximation is either circumscribed or inscribed by the cross-section of the sphere. A convergence study was then conducted to find the optimum number of modes required. The $\tan \delta$ of a quartz single crystal at room temperature ${ }^{22}$ is of the order of $2 \times 10^{-5}$. Krupka et al ${ }^{10}$ demonstrated that the temperature dependence of the losses in single crystal quartz are complex over the range 10-300 K but fairly constant over the $10-100 \mathrm{~K}$ range at around $1.2 \times 10^{-5}$. The permittivity normal to the axis, $\varepsilon_{t}=4.43$, only varies by $\pm 0.06 \%$ over the same temperature range. These data for single crystal quartz were used in the calculations. At room temperature, the resonant frequency of the quasi$\mathrm{TE}_{01 \delta}$ mode was measured at $9.3726 \mathrm{GHz}$ and had an unloaded $\mathrm{Q}$ factor of 61,877 . The relative 
permittivity $\varepsilon_{r}$, of the $\mathrm{MgO}$ sphere was calculated using the radial mode matching technique to be 9.64 .

The unloaded quality factor of the resonator is modelled by

$$
Q^{-1}=p_{d} \tan \delta+p_{s} \tan \delta+\frac{R_{S}}{G}
$$

where $p_{d}, \tan \delta_{d}$ and $p_{s}, \tan \delta_{s}$ are the electric filling factor and loss tangent of the dielectric sphere and the spacer respectively, $R_{S}$ is the surface resistance of the silver plated cavity and $G$ is its geometric factor. The electric filling factors can be calculated by considering the fraction of electric energy residing in the dielectric with respect to the total electric energy:

$$
p_{i}=\frac{\iiint_{V_{i}} \varepsilon_{r}|\mathbf{E}|^{2} d V}{\iiint_{V} \varepsilon_{r}(V)|\mathbf{E}|^{2} d V}
$$

For this case the filling factors for the $\mathrm{MgO}$ sphere and the quartz spacer were found to be $p_{d}=$ 0.8629 and $p_{s}=0.0476$, which shows that the $\mathrm{MgO}$ contains the bulk of the electric energy residing within the cavity. The geometric factor is evaluated from the expression:

$$
G=\frac{\iiint_{V} \mu_{0}|\mathbf{H}|^{2} d V}{\oiint_{S}\left|\mathbf{H}_{t}\right|^{2} d S}
$$

which involves volume and surface integrals of the magnetic fields. The geometric factor $G$ for the cavity was calculated to be 2373 . The room temperature surface resistivity $R_{S}$ was measured to be $29.5 \mathrm{~m} \Omega$, yielding conductor losses of $1.243 \times 10^{-5}$ which correspond to a conductor quality factor $Q_{c}$ of 80,441 . The loss tangent of the $\mathrm{MgO}$ sphere can then be calculated by rearranging (1):

$$
\tan \delta_{d}=\frac{1}{p_{d}}\left(\frac{1}{Q_{0}}-p_{s} \tan \delta_{s}-\frac{R_{S}}{G}\right)
$$

The permittivities of $\mathrm{MgO}$ where the grain boundary was aligned vertically or horizontally with respect to the cavity axis were measured as a function of temperature, and are almost identical as 
shown in Figure 3a. There was no significant difference in the relative permittivity of any of the spheres whether or not they contained a grain boundary.

The loss tangent, $\tan \delta$ for the spheres shown in Figure 3b. The orientation of the grain boundary, whether it is vertical or horizontal with respect to the cavity axis makes no difference to the measured loss. There is virtually no difference in the tan $\delta$ between any of the samples above $75 \mathrm{~K}$ - At around $45 \mathrm{~K}$ there is a pronounced peak in the tan $\delta$ for the bi-crystal which is absent for the single crystal. This peak is attributed to defect dipole relaxation of oxygen ions between oxygen vacancies. This phenomenon has been reported by Zuccaro et $\mathrm{al}^{23}$ in the case of oxygen vacancies in $\mathrm{LaAlO}_{3}$ caused by twin boundary defects.

The losses due to defect dipole Debye relaxation of oxygen ions at the grain boundary can be modelled using the following expression:

$$
\tan \delta=\frac{\Delta \varepsilon}{\varepsilon_{r} \varepsilon_{0}}\left(\frac{\omega \tau}{1+\omega^{2} \tau^{2}}\right)
$$

Where $\Delta \varepsilon=\eta N_{d} p^{2} / k_{B} T$, where $\eta$ is the field correction factor, $N_{d}$ is the defect density, and $p$ is the defect dipole moment. The thermally activated relaxation time $\tau$ is given by $\tau=\tau_{0} \exp \left(E / k_{B} T\right)$. A single Debye peak was fitted to the observed difference in loss tangent between a single crystal and bi-crystal but was found not to describe the data accurately. Twin Debye relaxation peaks were then fitted and a good agreement was found with experimental data as shown in Figure 4.

The total defect density was found to be $1.24 \times 10^{12} \mathrm{~cm}^{-3}$, with approximately equal contribution from the two peaks. The defect dipole activation energies for the peaks were found to be 14.9 and $18.3 \mathrm{meV}$ and the relaxation times 73.6 and $149.0 \mathrm{fs}$ respectively. These results suggest that dielectric losses at low temperatures are sensitive to very small concentrations of defects and that this measurement technique is capable of detecting and quantifying them.

The temperature dependence of the $\tan \delta$ indicates that there is a small contribution at low temperature which we interpret to be caused by Debye relaxation. The very low activation energies $(<20 \mathrm{meV})$ suggest that a collective motion of ions is responsible for the observed losses. 


\section{Conclusions}

The propensity for grain boundaries in polycrystalline ceramics to act as a sink for impurities, which do indeed have a deleterious effect on loss, is the main reason why grain boundaries have been held as the main contributors to dielectric loss. In the experiments carried out here on clean grain boundaries in $\mathrm{MgO}$ crystals, the influence on microwave loss is only seen at low temperatures. The temperature dependence of the $\tan \delta$ indicates that there is a small contribution at low temperature which we interpret to be caused by Debye relaxation.

The overall conclusion from this work is that grain boundaries have a very limited influence on the microwave dielectric loss. A single grain boundary in $\mathrm{MgO}$ has no effect on the microwave dielectric loss at room temperature and a very slight effect at low temperature (around $45 \mathrm{~K}$ ). Further, at room temperature, the difference in microwave loss between very pure single crystals and their polycrystalline counterparts is small and this has been shown to be the case in a number of ceramic dielectric materials including $\mathrm{Al}_{2} \mathrm{O}_{3}, \mathrm{TiO}_{2} \mathrm{MgO}$ and $\mathrm{LaAlO}_{3}{ }^{4,24,25,26}$. In these studies it was found that impurities and porosity were particularly deleterious to microwave loss.

\section{Figure Captions}

Fig 1 Dielectric resonator consisting of spherical $\mathrm{MgO}$ bi-crystal (diameter $=10 \mathrm{~mm}$ ), inside a cylindrical silver-plated cavity $(D=24.00 \mathrm{~mm}, L=16.10 \mathrm{~mm})$, placed upon single crystal quartz support ring ( $=5 \mathrm{~mm},=10 \mathrm{~mm}, l=4 \mathrm{~mm}$ ). The centre of the sphere is situated at a distance $h$ $=8.33 \mathrm{~mm}$ from the bottom of the cavity.

Figure 2 High Resolution TEM image of the grain boundary interface. 
Figure 3a Relative permittivity of $\mathrm{MgO}$ measured over $25-250 \mathrm{~K}$ temperature range for vertical $(\mathrm{V})$ and horizontal $(\mathrm{H})$ grain boundary alignments.

Figure 3b. Measured tan $\delta$ for vertical and horizontal alignments of the single grain boundary in a spherical $\mathrm{MgO}$ bi-crystal together with a grain boundary free $\mathrm{MgO}$ sphere. The small peak in $\tan \delta$ for samples containing a grain boundary at around $45 \mathrm{~K}$ which is thought to be due to defect dipole relaxation of oxygen vacancies

Figure 4. Difference in loss tangent $\Delta \tan \delta$ between a $\mathrm{MgO}$ bi-crystal containing a single grain boundary and a grain boundary free $\mathrm{MgO}$ single crystal. Both samples were spherical and resonated at $9.37 \mathrm{GHz}$. Multiple Debye peaks were fitted to the observed peak.

\section{References}

${ }^{1}$ Cottrell, A.H. Strong Solids. Proc. Royal Soc. A282, 2-9 (1964).

${ }^{2}$ Griffith, A.A. The phenomenon of rupture and flow in solids. Phil. Trans. Roy. Soc. 221, 163198 (1920).

${ }^{3}$ Alford, N.M., Birchall, J.D. \& Kendall, K. High-strength ceramics through colloidal control to remove defects. Nature 330, 51-55 (1987).

${ }^{4}$ Alford, N.M. \& Penn, S.J. Sintered alumina with low dielectric loss. Journal of Applied Physics 80, 5895-5898 (1996).

${ }^{5}$ Lord Rayleigh. Phil. Mag. S. 5 43, 125-132 (1897).

${ }^{6}$ Debye, P. Lichtdruck auf Kugeln von bliebigem Material. Annalen der Physik 30, 57-136 (1909).

${ }^{7}$ Braginsky, V.B. \& Ilchenko, V.S. Experimental observation of fundamental microwave absorption in high-quality dielectric crystals. Physics Letters A 120, 300-305 (1987).

${ }^{8}$ Gurevich, V.L. \& Tagantsev, A.K. Intrinsic dielectric loss in crystals. Advances in Physics 40, 719-767 (1991).

${ }^{9}$ Sparks, M., King, D.F., \& Mills, D.L. Phys. Rev. B 26, 6987 (1981).

${ }^{10}$ Krupka, J., Derzakowski, K., Tobar, M., Hartnett, J. \& Geyer, R. G. Complex permittivity of some ultralow loss dielectric crystals at cryogenic temperatures. Measurement Science \& Technology 10, 387-392 (1999).

${ }^{11}$ Kim, I.-T., Kim, Y. \& Chung, S.J. Order-disorder transition and microwave dielectric properties of $\mathrm{Ba}(\mathrm{NiNb}) \mathrm{O}$ ceramics. Japanese Journal of Applied Physics 34, 4096-4103 (1995).

${ }^{12}$ Ichinose N. and Shimada T., Effect of grain size and secondary phase on microwave dielectric properties of $\mathrm{Ba}\left(\mathrm{Mg}_{1 / 3} \mathrm{Ta}_{2 / 3}\right) \mathrm{O}_{3}$ and $\mathrm{Ba}\left([\mathrm{Mg}, \mathrm{Zn}]_{1 / 3} \mathrm{Ta}_{2 / 3}\right) \mathrm{O}_{3}$ systems. J. Europ. Ceram. Soc. 26, 1755-1759 (2006).

${ }^{13}$ Kim, E.S. \& Yoon, K.H. Effect of nickel on microwave dielectric properties of Ba(MgTa)O. Journal of Materials Science 29, 830-834 (1994). 
${ }^{14}$ Prasad, K. V.R., Raju, A.R. \& Varma, K. B.R. Grain size effects on the dielectric properties of ferroelectric BiVO ceramics. Journal of Materials Science 29, 2691-2696 (1994).

${ }^{15}$ Chen, X.M., Liu, D., Hou, R.Z. \& Hu, X. Microstructures and Microwace Dielectric Characteristics of $\mathrm{Ca}(\mathrm{ZnNb}) \mathrm{O}$ ceramics. Journal of the American Ceramamic Society 87, 22082212 (2004).

${ }^{16}$ Kucheiko, S., Kim, H.-J., Yoon, S.-J. \& Jung, H.-J. Effect of ZnO additive on the microstructure and microwave dielectric properties of $\mathrm{CaTi}(\mathrm{FeNb}) \mathrm{O}$ ceramics. Japanese Journal of Applied Physics 36, 198-202 (1997).

${ }^{17}$ McNeal, M.P., Jang, S.-J. \& Newnham, R.E. The effect of grain and particle size on the microwave properties of barium titanate BaTiO. Journal of Applied Physics 83, 15 (1998).

${ }^{18}$ Schlomann, E. "Dielectric Losses in ionic crystals with disordered charge distributions" Phys Rev B 35 (2a) p A413 - A419 (1964)

${ }^{19}$ Wang, C. \& Zaki, K.A. Generalized multilayer anisotropic dielectric resonators. IEEE Trans. Micro. Theory Tech. 48, 60 (2000).

${ }^{20}$ Kajfez, D. \& Guillon, P. Dielectric Resonators (Artech House, 1986).

${ }^{21}$ Hui, W.K. \& Wolff, I. A Multicomposite, Multilayered Cylindrical Dielectric Resonator for Application in MMIC's. IEE-MTT 42, 361 (1994).

${ }^{22}$ Zychowicza, T., Krupka, J. \& Tobar, M.E. Whispering gallery modes in hollow spherical dielectric resonators. J. Eur. Ceram. Soc. 26, 2193-2194 (2006).

${ }^{23}$ Claudio Zuccaro, Michael Winter, Norbert Klein, and Knut Urban "Microwave absorption in single crystals of lanthanum aluminate" Journal of Applied Physics -- December 1, 1997 -Volume 82, Issue 11, pp. 5695-5704

24 Jonathan D. Breeze, Xavi Aupi and Neil McN. Alford. "Ultra-Low Loss Polycrystalline Alumina” Appl Phys Lett vol 81 p 5021 (2002)

${ }^{25}$ Templeton Alan Templeton, Xiaoru Wang, Stuart J Penn, Stephen J Webb, Lesley F Cohen and Neil McN Alford "Microwave Dielectric Loss of Titanium Oxide" J Amer Ceram Soc 83 (1) 95-100 (2000).

${ }^{26}$ N.McN. Alford, J Breeze, X Wang, SJ Penn, S Dalla, SJ Webb, N Ljepojevic and X Aupi. "Dielectric Loss of oxide single crystals and polycrystalline analogues from 10 to $320 \mathrm{~K}$ " J European Ceramic Society vol 21 pp 2605-2611 (2001) 
Fig 1

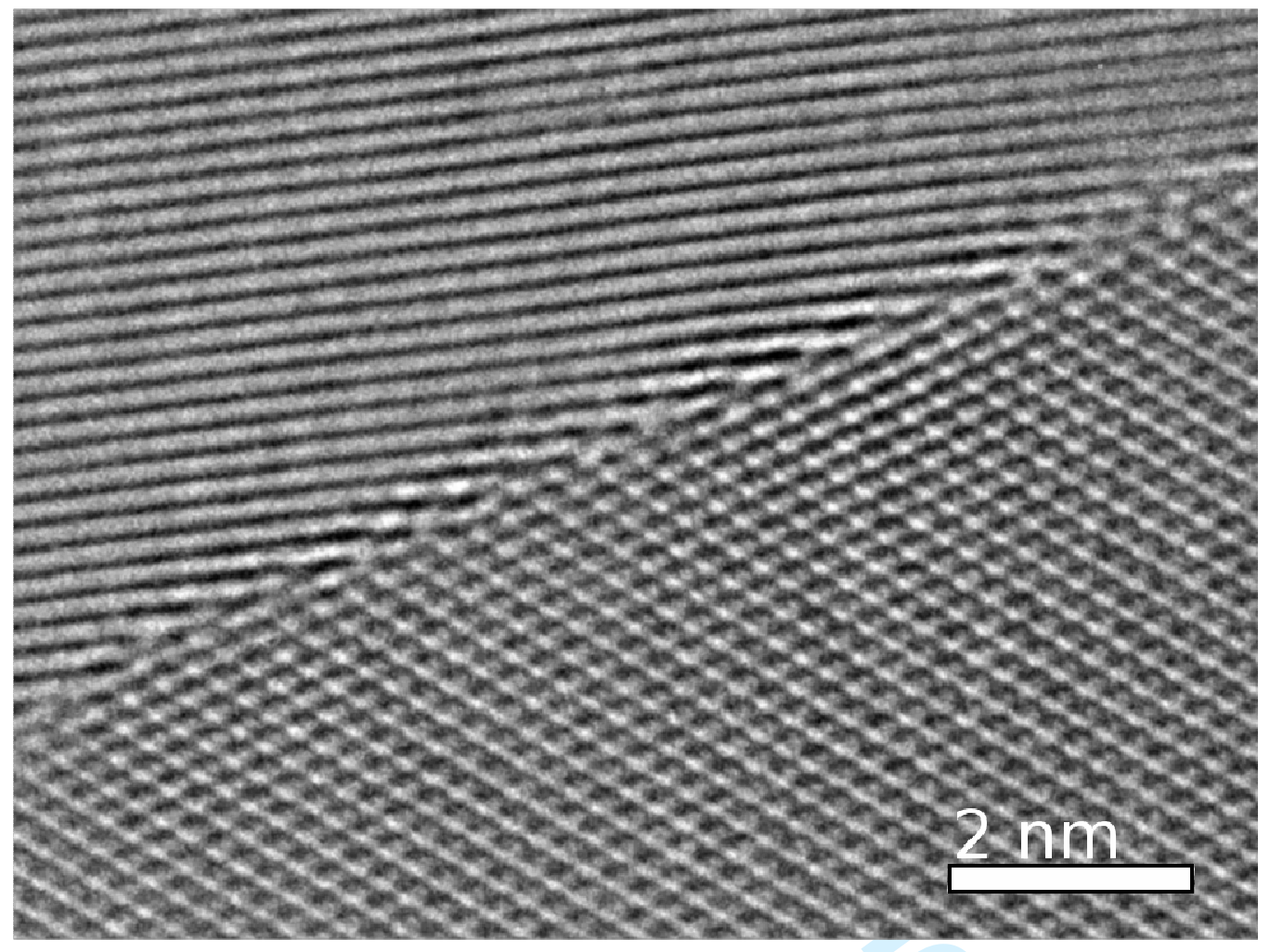


Fig 2

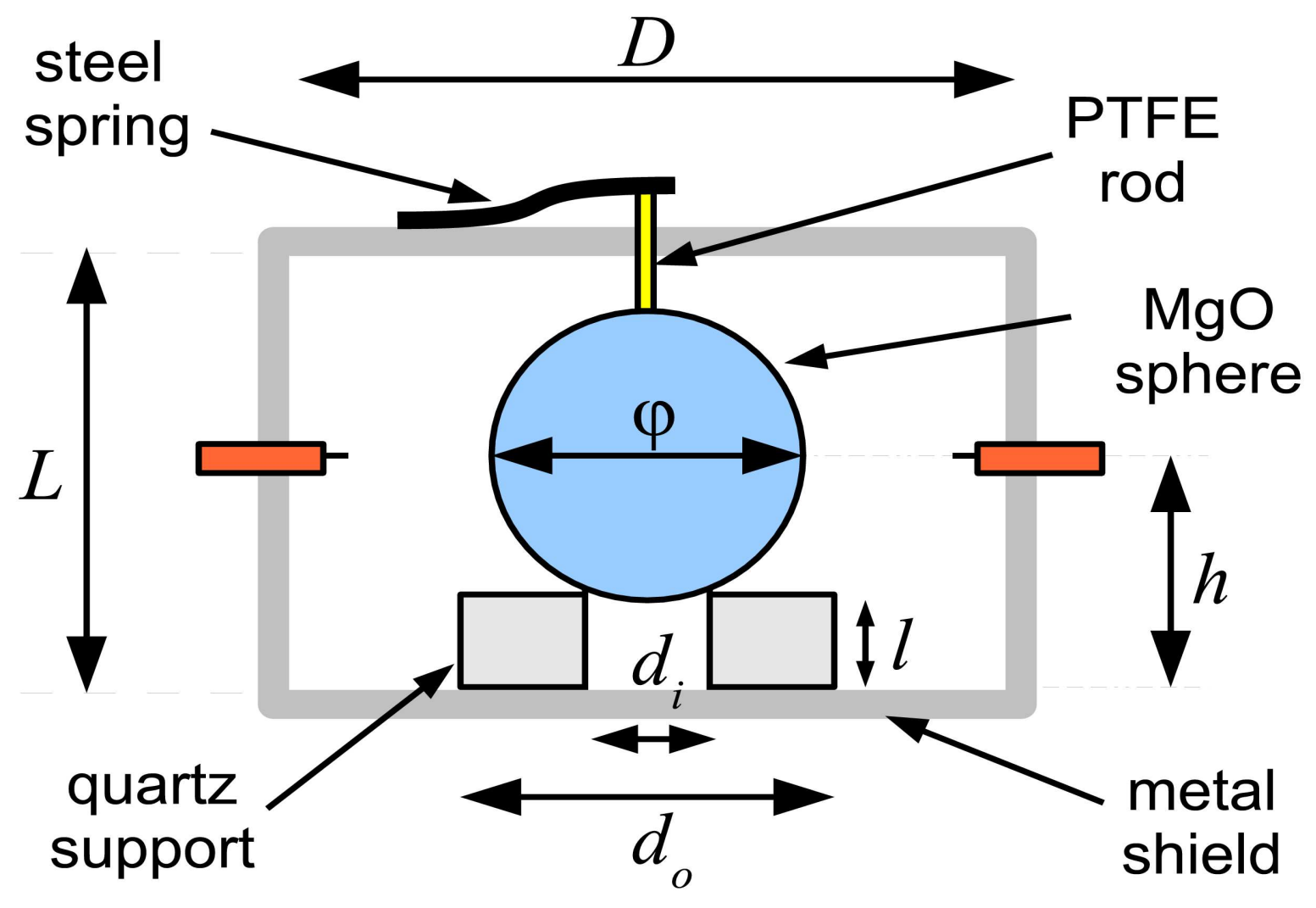


fig $3 a$

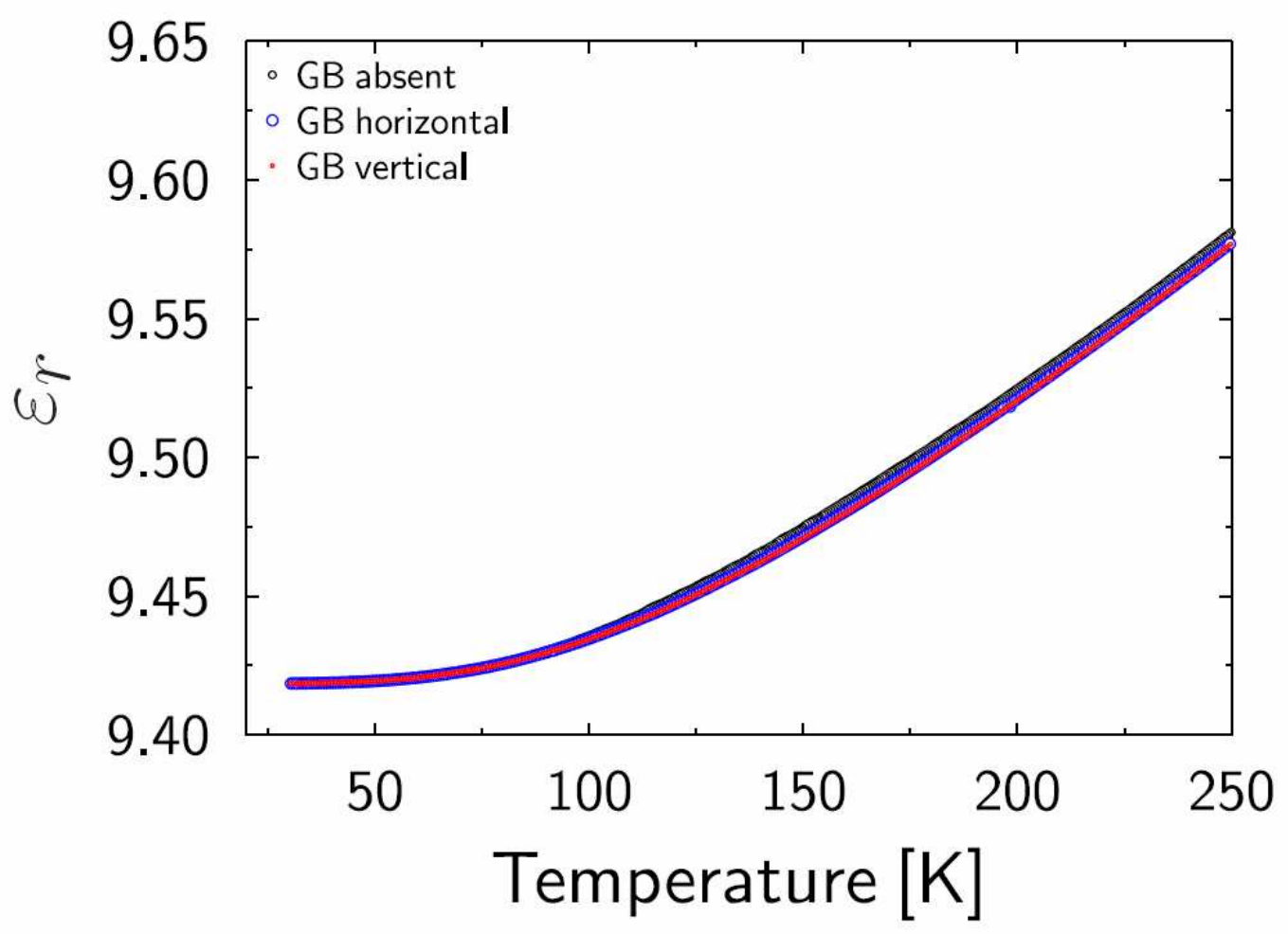


fig $3 b$

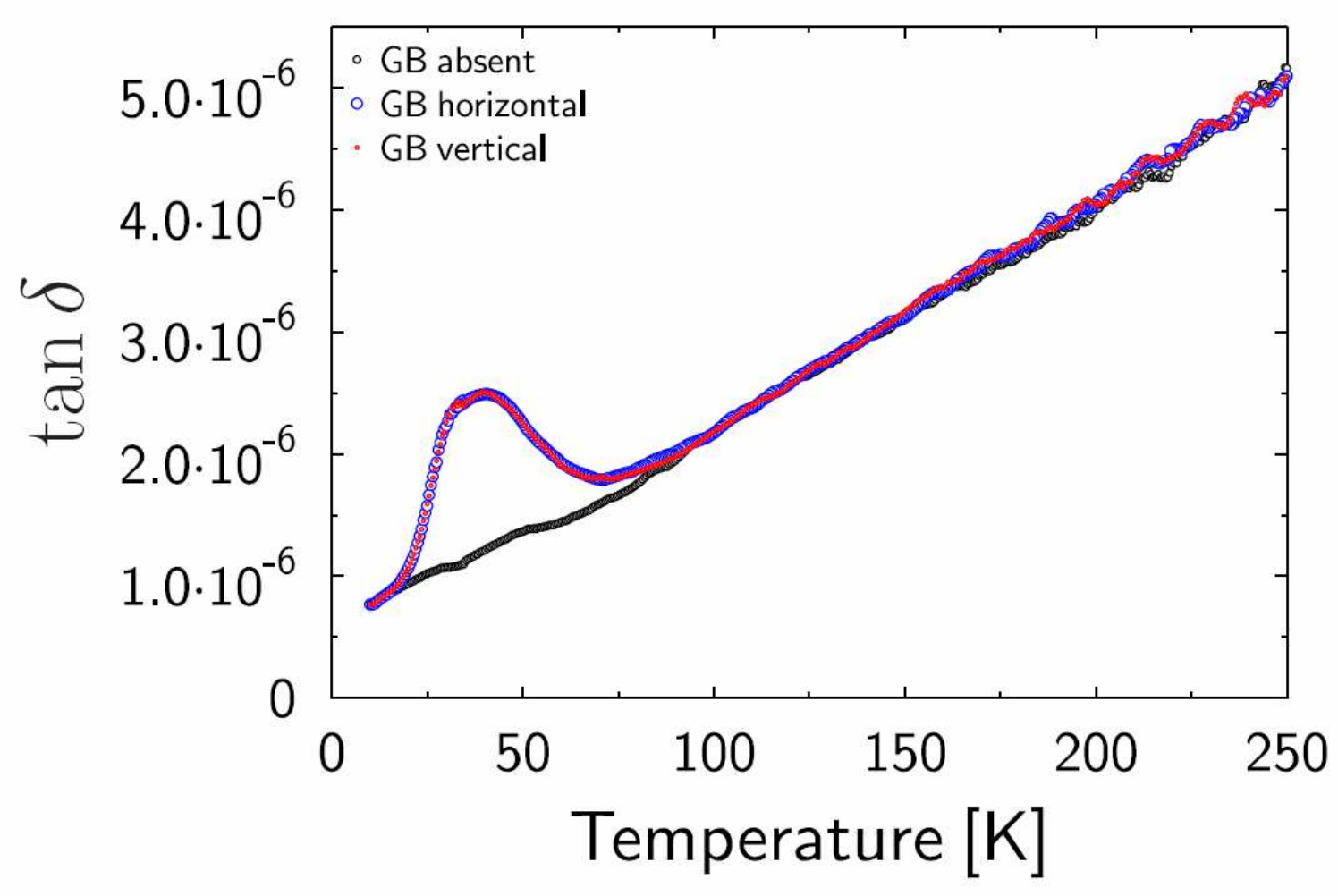


fig 4

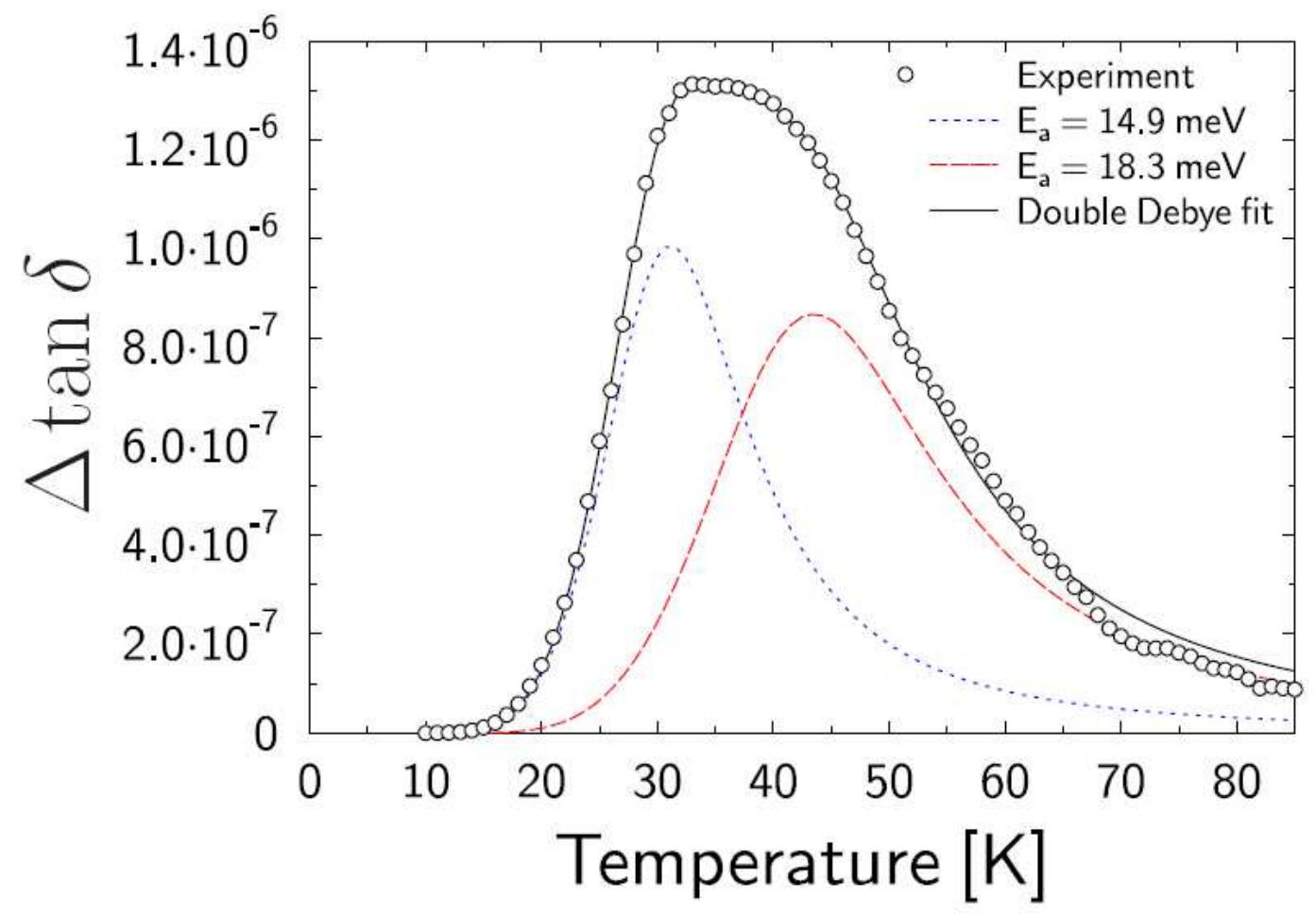

